Check for updates

Cite this: J. Mater. Chem. C, 2021 , 9, 10794

\section{Impact of chemical modifications on the luminescence properties of organic neutral radical emitters $\dagger+$}

\author{
Eunkyung Cho, (D) Veaceslav Coropceanu (D) * and Jean-Luc Brédas (D) *
}

Received 12th April 2021, Accepted 17th May 2021

DOI: 10.1039/d1tc01702k

rsc.li/materials-c

\begin{abstract}
Neutral organic radicals have recently attracted great attention as promising luminescent materials, which is a consequence of their strong doublet emission properties. Recent investigations have indicated that even minor chemical modifications can have a significant impact on the luminescence properties of these radical emitters. Here, we performed long range-corrected density functional theory calculations to evaluate how chemical modifications affect the electronic properties and radiative and nonradiative decay rates in a series of tris(2,4,6-trichlorophenyl)methyl-pyridoindole (TTM-xPyID) radicals vs. the TTM-carbazole radical. We find that the lowest excited state of these radical emitters has a charge-transfer (CT) character and its energy blue-shifts upon chemical modification from carbazole to XPyID, in agreement with experiment. An analysis of the transition dipole moments shows that hybridization between the CT and ground states of the TTM-xPyID radicals plays a dominant role in the radiative decay rates. On the other hand, hybridization between the $\mathrm{CT}$ state and the lowest localexcitation (LE) state on the TTM radical core has a significant contribution to the nonradiative rates in most of TTM-xPyID radicals, while it has only a minor influence on that rate in the TTM-carbazole radical. Our results underline that the hybridization of the CT state with both ground state and LE state can substantially influence the radiative and non-radiative rates in TTM-based radicals. Also, we show that the relative contributions of these two hybridization pathways depend in a subtle way on the properties of the donor fragment, such as its ionization potential and the intramolecular relaxion energy associated with its oxidation process. Finally, we propose a new design strategy to achieve high values of photoluminescence quantum yield in radicals with a donor-acceptor structural motif.
\end{abstract}

\section{Introduction}

Stable neutral organic radicals have received a great deal of interest due to their possible applications in a variety of fields including optoelectronics, spintronics, magnets, and quantum information technologies. ${ }^{1-8}$ In particular, significant advances have been recently achieved in the field of organic lightemitting diodes (OLEDs). ${ }^{9-15}$ These radicals have an openshell electronic configuration and in many instances both the ground state $\left(D_{0}\right)$ and the first-excited state $\left(D_{1}\right)$ have a spin-doublet character. ${ }^{16-19}$ This is in marked contrast with conventional closedshell organic emitters where the emissive singlet state is located above the first excited triplet state; as a result, $\sim 75 \%$ of the electrically generated excitons are triplet excitons whose emission

Department of Chemistry and Biochemistry, The Univeszrsity of Arizona, Tucson, Arizona 85721-0088, USA.E-mail: coropceanu@arizona.edu, jlbredas@arizona.edu $\dagger$ Electronic supplementary information (ESI) available. See DOI: 10.1039/d1tc01702k ‡ Dedicated to Professor Concepció Rovira and Professor Jaume Veciana, in honor of their seminal contributions to the fields of molecular electronics and magnetism. is spin-restricted. In their majority, the neutral radical emitters that are currently investigated have a donor-acceptor (D-A $\mathrm{A}^{\bullet}$ chemical structure, in which an electron-poor acceptor radical $\left(\mathrm{A}^{\bullet}\right)$ core, such as a stable tris(2,4,6-trichlorophenyl)methyl (TTM) or perchlorotriphenylmethyl (PTM) radical, is covalently linked to an electron-rich donor group (D), such as a carbazole $(\mathrm{Cz})$ derivative. As a consequence, the $\mathrm{D}_{1}$ state has a charge-transfer nature ${ }^{9,14,15,17,20,21}$ and the radiative and nonradiative transitions back to $\mathrm{D}_{0}$ can be viewed as a back-electron transfer from the $\mathrm{A}^{-}$ moiety to the $\mathrm{D}^{+}$moiety. ${ }^{10,19}$

In 2015, Peng et al. ${ }^{11}$ reported the first viable OLED based on the red TTM-1Cz radical emitter, ${ }^{9}$ which consists of a combination of the TTM radical with carbazole (see Fig. 1). TTM-1Cz displays a photoluminescence quantum yield (PLQY) of 53\% in cyclohexane and high photostability, which motivated further investigations of organic radicals for OLED applications and led to the active development of a number of new radical emitters. ${ }^{14,15,22,23}$ The most efficient radical emitter OLEDs reported so far are those based on TTM-3NCz (9-(naphthalene-2-yl)-9H-carbazole), which have external quantum efficiencies (EQEs) of up to $27 \%{ }^{14}$ 


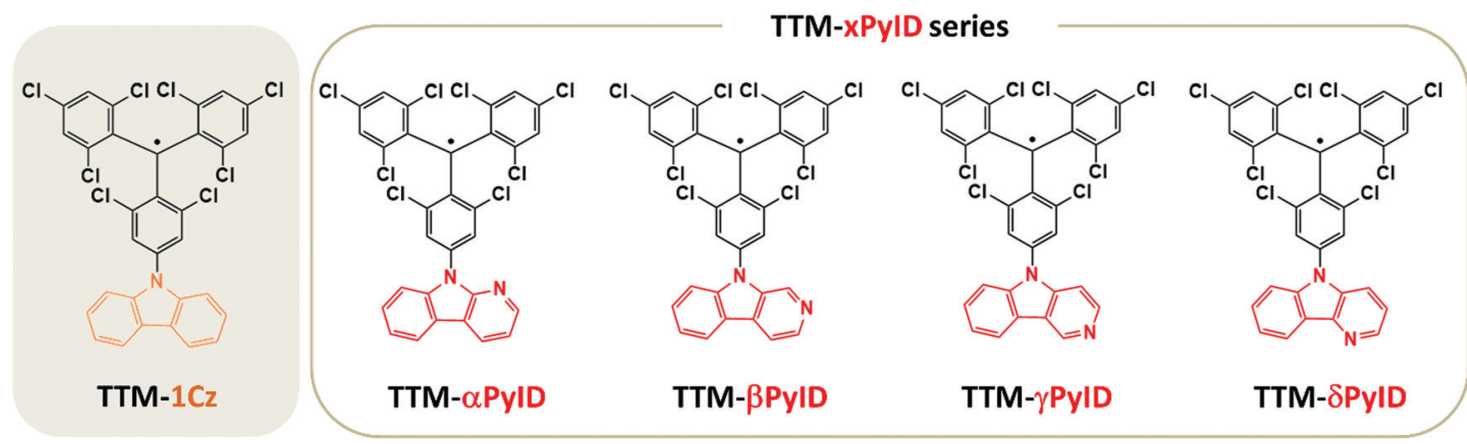

Fig. 1 Chemical structures of TTM-1Cz and TTM-xPyID $(x=\alpha, \beta, \gamma$ and $\delta)$.

In $\mathrm{D}-\mathrm{A}^{\bullet}$ radical emitters, the hybridization between the $\mathrm{D}_{1}$ charge-transfer (CT) state and the low-energy local-excitation (LE) states can play a significant role on the emissive properties. For instance, we have reported that in PTM-TAA radicals (where TAA denotes a substituted triarylamine), CT-LE hybridization significantly increases the non-radiative decay rates, ${ }^{19}$ while Abdurahman et al. suggested recently that in TTM-xPyID radicals (where PyID stands for pyridoindole, see Fig. 1), CT-LE hybridization contributes appreciably to the radiative decay of the CT states. ${ }^{17}$ To shed more light on the role of CT-LE hybridization on the radical emissive properties, we describe here the electronic structures and radiative and nonradiative decay rates of a series of TTM-xPyID radicals, as well as of $\mathrm{TTM}-1 \mathrm{Cz}$ for the sake of comparison.

\section{Computational methods}

In order to determine the ground-state electronic structures of the radical emitters, we performed density functional theory (DFT) calculations using the screened range-separated hybrid (SRSH) functional LC- $\omega$ hPBE with $20 \%$ Hartree Fock exchange and the $6-31 G^{* *}$ basis set. ${ }^{24}$ In our recent work on PTM-TAA radicals, we found that this computational approach provides results that are in very good agreement with experiment. ${ }^{19}$ The range-separation parameters $(\omega)$ were optimized for each radical. We used a dielectric constant $\varepsilon=2$ in the SRSH calculation to account for the screening of electrostatic electron-electron interactions due to the surroundings. The excited-state properties were obtained at the time-dependent DFT level within the TammDancoff approximation (TDA-TDDFT). ${ }^{25}$ Natural transition orbital (NTO) analyses were carried out to characterize the nature of the excited states. All DFT/TDDFT calculations were performed with the Gaussian 16 program. $^{26}$

Within the two-state model, the radiative decay rates from the CT states were obtained by means of eqn (1): ${ }^{27}$

$$
k_{\mathrm{r}(\mathrm{CT})}=\frac{E_{\mathrm{CT}}^{3} f(n)}{3 \pi \varepsilon_{0} \hbar^{4} c^{3}} \mu_{\mathrm{CT}}^{2}
$$

where $\varepsilon_{0}, c, E_{\mathrm{CT}}, f(n)$, and $\mu_{\mathrm{CT}}$ denote the vacuum permittivity, speed of light, CT $\left(\mathrm{D}_{1}\right)$ state energy, local-field correction factor, and transition dipole moment between ground state and CT state, respectively. The local-field correction factor, $f(n)=\frac{n\left(n^{2}+2\right)^{2}}{9}$, accounts for the local electric field effect and is considered according to the Lorentz virtual cavity model; ${ }^{28}$ the value of $\mathrm{n}$ was set to 1.43 (corresponding to the refractive index of cyclohexane).

The nonradiative decay rates from the CT states were calculated in the framework of the Marcus-Levich-Jortner approach: ${ }^{29}$

$$
\begin{aligned}
k_{\mathrm{nr}}^{\mathrm{CT}-\mathrm{GS}}= & \frac{2 \pi}{\hbar}\left|V_{\mathrm{CT}-\mathrm{GS}}\right|^{2} \frac{1}{\sqrt{4 \pi \lambda_{\mathrm{c}} k_{\mathrm{B}} T}} \sum_{n=0}^{\infty} e^{-S_{\mathrm{qm}}} \frac{S_{\mathrm{qm}}^{n}}{n !} \\
& \times \exp \left[-\frac{\left(n \hbar \omega_{\mathrm{qm}}+\lambda_{\mathrm{c}}-E_{\mathrm{CT}}^{\mathrm{a}}\right)^{2}}{4 \lambda_{\mathrm{c}} k_{\mathrm{B}} T}\right]
\end{aligned}
$$

where $V_{\mathrm{CT}-\mathrm{GS}}$ and $E_{\mathrm{CT}}^{\mathrm{a}}$ represent the electronic coupling between the CT and ground states and the adiabatic CT energy, respectively; $S_{\mathrm{qm}}$ and $\omega_{\mathrm{qm}}$ are the effective Huang-Rhys factor and vibrational frequency, respectively, which define the quantum contribution $\lambda_{\mathrm{qm}}=S_{\mathrm{qm}} \omega_{\mathrm{qm}}$, to the reorganization energy due to high-frequency vibrational modes; $\lambda_{\mathrm{c}}$ denotes the classical part of the reorganization energy due to low-frequency modes. The total reorganization energy $\lambda\left(\lambda=\lambda_{\mathrm{c}}+\lambda_{\mathrm{qm}}\right)$ is obtained from the adiabatic potential energy surfaces of the $D_{0}$ and $D_{1}$ states. ${ }^{30}$ The electronic couplings between the CT and ground states, and between the CT and LE states were determined from the generalized Mulliken-Hush approach $^{31}$ as implemented in the Q-Chem 5 package. ${ }^{32}$ The CT components in each doublet state of the radicals were evaluated by calculating the changes in the $\mathrm{D}$ and $\mathrm{A}$ contributions to the molecular orbitals involved in the given electronic transitions; this analysis was carried out with the GaussSum software; ${ }^{33}$ the $\mathrm{Cz}$ or xPyID unit is assigned as the donor fragment while the TTM radical is defined as the acceptor fragment.

In order to account for the hybridization between the CT and LE states and consider its impact on the non-radiative rates in the TTM-1Cz and TTM-xPyID radicals, we followed the approach we described in our previous work: ${ }^{19,34}$

$$
\begin{gathered}
k_{\mathrm{nr}}=\left(1-f_{\mathrm{CT}-\mathrm{LE}}^{2}\right) k_{\mathrm{nr}}^{\mathrm{CT}-\mathrm{GS}}+f_{\mathrm{CT}-\mathrm{LE}}^{2} k_{\mathrm{nr}}^{\mathrm{LE}-\mathrm{GS}} \\
f_{\mathrm{CT}-\mathrm{LE}}=\left(\frac{V_{\mathrm{CT}-\mathrm{LE}}}{\Delta E_{\mathrm{CT}-\mathrm{LE}}}\right) /\left[\left(1+\left(\frac{V_{\mathrm{CT}-\mathrm{LE}}}{\Delta E_{\mathrm{CT}-\mathrm{LE}}}\right)^{2}\right)\right]^{1 / 2}
\end{gathered}
$$

Here, $k_{\mathrm{nr}}^{\mathrm{LE}-\mathrm{GS}}$ represents the non-radiative rate for a transition from an LE state (i.e., the first excited state localized on the radical core) to the ground state (GS) and $f_{\mathrm{CT}-\mathrm{LE}}$ represents the 
degree of hybridization between the CT and LE states. In the calculations we report below, we account only for the nonradiative transition from the first excited state of the radical core by taking $k_{\mathrm{nr}}^{\mathrm{LE}-\mathrm{GS}}$ equal to the experimental value of $1.75 \times$ $10^{8} \mathrm{~s}^{-1}$ measured for the TTM radical. ${ }^{17}$ We note that a similar approach can be used to account for the effect of CT-LE hybridization on the radiative transitions. ${ }^{34,35}$

\section{Results and discussion}

Fig. 2 displays the energies of the frontier molecular orbitals involved in the lowest ten excited doublet $\left(D_{1}-D_{10}\right)$ states and the first excited quartet $\left(\mathrm{Q}_{1}\right)$ state of the TTM-based radicals, as computed at the ground-state geometry. Although the singly occupied molecular orbital (SOMO) level of the TTM moiety is located below the highest doubly occupied molecular orbital (HOMO) levels of the $\mathrm{Cz}$ and xPyID fragments (see Fig. S1, $\mathrm{ESI} \dagger$ ), all $\mathrm{D}-\mathrm{A}^{\bullet}$ radicals investigated here have an Aufbau electronic configuration, i.e., the SOMO level in the whole molecule is located above the doubly occupied HOMO level. In comparison to TTM- $1 \mathrm{Cz}$, the introduction of an additional nitrogen into carbazole to form pyridoindole does not result in any substantial change in the HOMO, SOMO, and lowest fully unoccupied molecular orbital (LUMO) energies (Fig. 2a). For all TTM-based radicals, the SOMO is delocalized over the entire molecule while the HOMO and LUMO are mainly localized on the donor moiety and the TTM moiety, respectively (see Fig. S2, ESI $\dagger$ ).

As seen from Fig. $2 \mathrm{~b}$, the $\mathrm{D}_{1}$ energy increases when replacing $\mathrm{Cz}$ with xPyID. This blue-shift in the $\mathrm{D}_{1}$ absorption band agrees well with the experimental data. The variation in $\mathrm{D}_{1}$ energy as a function of the nitrogen position within xPyID is small. Also, the $\mathrm{D}_{2}$ states in the TTM-xPyID radicals correspond to the first excited state localized on the TTM moiety (we label them as $\mathrm{LE}_{1}$ states) and their energies remain nearly constant along the series. We note that, in the case of TTM-1Cz, the first TTM local-excitation state corresponds to the $\mathrm{D}_{3}$ state. There are two high-energy LE states in the TTM-xPyID radicals, $\mathrm{D}_{8}$ and either $\mathrm{D}_{9}$ or $\mathrm{D}_{10}$ (which we label as $\mathrm{LE}_{\mathrm{r} 1}$ and $\mathrm{LE}_{\mathrm{r} 2}$ states), which are related to local excitations within the TTM core and have large oscillator strengths (see Tables S1 and S2, ESI $\dagger$ ); their energies are also nearly constant in going from $\mathrm{Cz}$ to xPyID.

An analysis of the natural transition orbitals (NTOs, see Fig. 3) indicates that the $\mathrm{D}_{1}$ state in the TTM-1Cz and TTM-xPyID radicals has a CT nature, coming from an electron transfer from the $\mathrm{Cz}$ or xPyID donor to the TTM acceptor (which roughly corresponds to a HOMO-to-LUMO transition). The NTOs also confirm that the $\mathrm{D}_{2}$ $\left(\mathrm{LE}_{1}\right), \mathrm{LE}_{\mathrm{r} 1}$, and $\mathrm{LEr}_{2}$ states have a dominant $\mathrm{LE}$ character. These results are further supported by the evaluations of the CT components in each state, see Table 1.

The TD-DFT calculated radiative rates for the transition from the CT $\left(D_{1}\right)$ state back to the ground $\left(D_{0}\right)$ state are listed in Table 2, along the experimental rates. Overall, the DFT values compare very well with the experimental values, although in the case of TTM- $\beta$ PyID and TTM- $\delta$ PyID, the DFT estimates are somewhat smaller than the measured values.

In agreement with experiment, the computed radiative rates for the TTM-1Cz and TTM-xPyID radicals are about 3 to 6 times as large as the value of $3.5 \times 10^{6} \mathrm{~s}^{-1}$ measured for the TTM radical core. ${ }^{17}$ The increase in the radiative rate upon addition of a donor to the TTM moiety is thus related to the formation of a CT state as the first excited state of the D-A ${ }^{\bullet}$ systems. ${ }^{10,15,19}$ The question then becomes what does determine the value of the $\mu_{\mathrm{CT}}$ transition dipole and make it larger than $\mu_{\mathrm{D} 1}$ in TTM? We recall that in the diabatic approximation (where no coupling is considered between the CT state and any other state), $\mu_{\mathrm{CT}}$ is zero. Therefore, the actual value of $\mu_{\mathrm{CT}}$ comes from the hybridization of the CT state with the ground state (GS) and local excited states of the TTM and D moieties. Our TD-DFT calculations show that in all systems $\mu_{\mathrm{CT}}$ is parallel to the vector connecting the centers of the $\mathrm{D}$ and $\mathrm{A}^{\bullet}$ units (see Fig. S3, ESI $\dagger$ ); this is a strong indication that $\mu_{\mathrm{CT}}$ is determined by the coupling of the CT state with the ground state. ${ }^{29}$ In contrast, Abdurahman et al. ${ }^{17}$ suggested recently that $\mu_{\mathrm{CT}}$ in TTM-xPyID radicals is defined by the hybridization of the

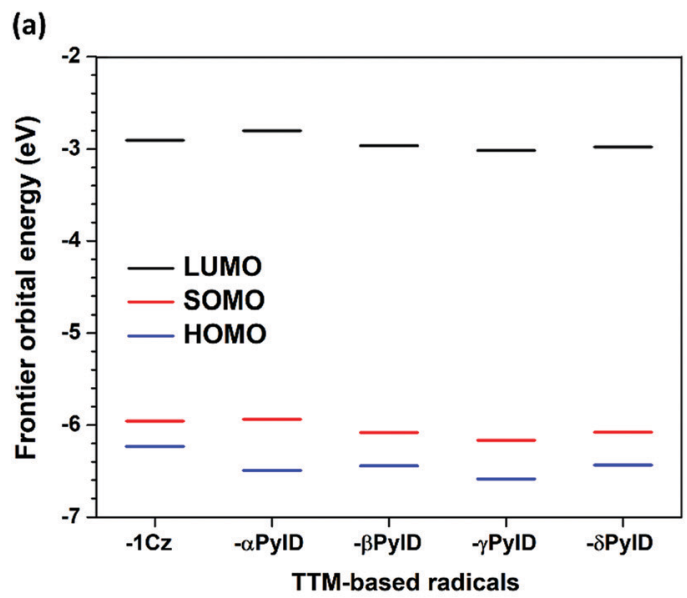

(b)

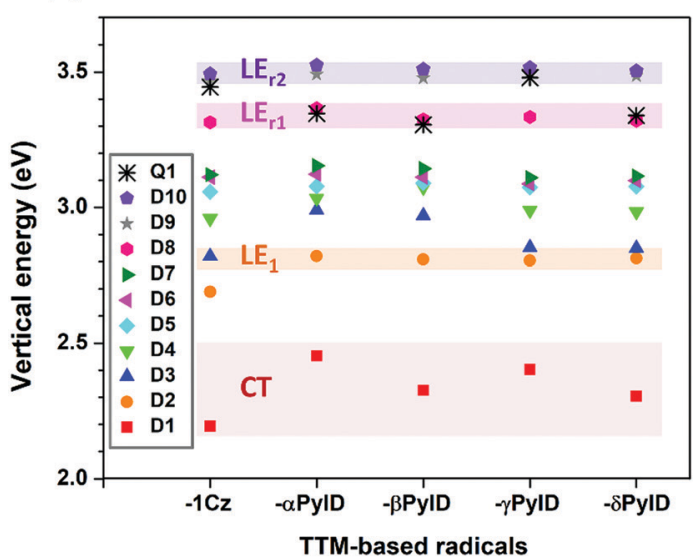

Fig. 2 Energies of (a) the LUMO, SOMO, and HOMO levels and (b) the doublet $\left(D_{1}-D_{10}\right)$ and quartet $\left(Q_{1}\right)$ states of the $T T M-1 C z$ and $T T M-x P y I D$ radicals; $C T, L E_{1}, L E_{r 1}$, and $L E_{r 2}$ represent the $D_{1}, D_{2}\left(D_{3}\right.$ for $\left.T T M-1 C z\right), D_{8}$, and $D_{10}\left(D_{9}\right.$ for $\left.T T M-\alpha P y I D\right)$ states of the radicals, respectively. All the molecular geometries are the optimized ground-state geometries. 

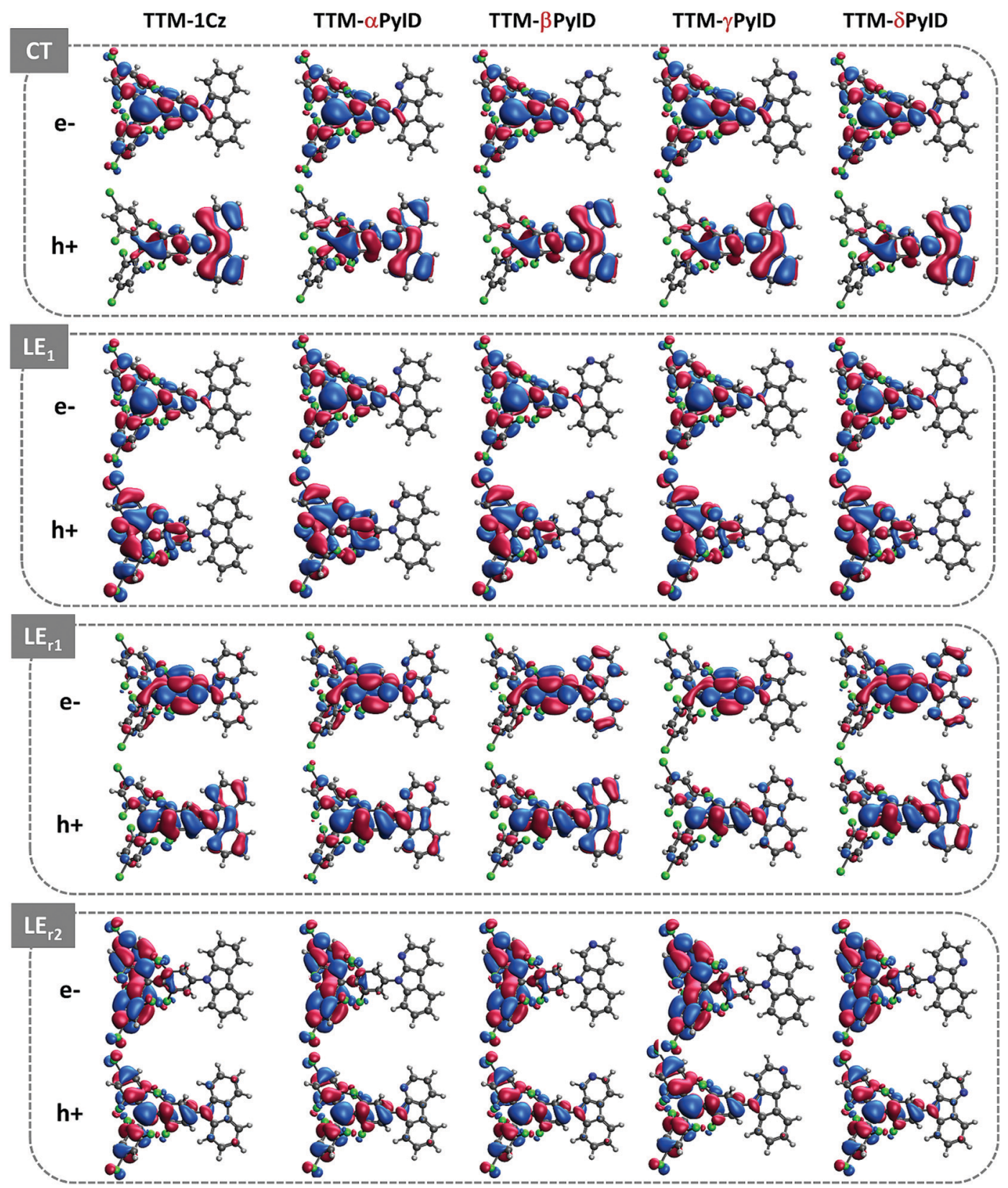

Fig. 3 Electron and hole natural transition orbitals (NTOs) in the $C T, L E_{1}, L E_{r 1}$, and $L E_{r 2}$ states of the TTM-1Cz and TTM-xPyID radicals.

Table 1 Calculated charge-transfer (CT) components (in \%) in the CT, LE 1 , $L E_{r 1}$, and $L E_{r 2}$ states of the TTM-1Cz and TTM-xPyID radicals

\begin{tabular}{llllll}
\hline State & TTM-1Cz & TTM- $\alpha$ PyID & TTM- $\beta$ PyID & TTM- $\gamma$ PyID & TTM- $\delta$ PyID \\
\hline CT & 82 & 70 & 78 & 78 & 79 \\
$\mathrm{LE}_{1}$ & 9 & 5 & 5 & 3 & 18 \\
$\mathrm{LE}_{\mathrm{r} 1}$ & 23 & 24 & 2 & 26 & 19 \\
$\mathrm{LE}_{\mathrm{r} 2}$ & 36 & 14 & 28 & 28 & 31
\end{tabular}

CT state with the TTM $\mathrm{D}_{2}$ state (which corresponds to an intensity borrowing effect). Our calculations show that in TTM the transitions from the lowest six excited states to the ground state have relatively small transition dipole moments. However, the transition dipole moments for the transitions from the
Table 2 TD-DFT and experimental values of the radiative decay rates from the CT state in TTM-1Cz and TTM-xPyID radicals

\begin{tabular}{lll}
\hline State & $k_{\mathrm{r}} \times 10^{7} \mathrm{~s}^{-1}(\mathrm{TD}-\mathrm{DFT})$ & $k_{\mathrm{r}} \times 10^{7} \mathrm{~s}^{-1}(\exp )^{a}$ \\
\hline TTM-1Cz & 1.14 & 1.28 \\
TTM- $\alpha$ PyID & 1.45 & 1.54 \\
TTM- $\beta$ PyID & 1.22 & 2.24 \\
TTM- $\gamma$ PyID & 1.18 & 1.00 \\
TTM- $\delta$ PyID & 1.23 & 2.08 \\
${ }^{a}$ Measured in cyclohexane. ${ }^{17}$ & \\
\hline
\end{tabular}

$\mathrm{D}_{7}$ and $\mathrm{D}_{8}$ states to the ground state are significant $(4.1 \mathrm{D})$, see Table S1 (ESI $\dagger$ ). These two TTM states, as discussed above, correspond to $\mathrm{D}_{8}\left(\mathrm{LE}_{\mathrm{r} 1}\right)$ and either $\mathrm{D}_{9}$ or $\mathrm{D}_{10}\left(\mathrm{LE}_{\mathrm{r} 2}\right)$ in TTM-1Cz 
and TTM-xPyID (see Table S2, ESI $\dagger$ ). As seen from Table 1, $\mathrm{LE}_{\mathrm{r} 1}$ and $\mathrm{LE}_{\mathrm{r} 2}$ have a significant $\mathrm{CT}$ component, which is consistent with the observed decrease in the intensity of the absorption band at about $3.2 \mathrm{eV}$, corresponding to these transitions, when going from TTM to TTM-1Cz. As such, these LE states of the TTM moiety could in principle contribute to $\mu_{\mathrm{CT}}$.

Next, in order to shed more light on this issue, we study in more detail the CT-GS and CT-LE contributions to $\mu_{\mathrm{CT}}$. The related electronic couplings are collected in Table 3. In the perturbation limit, the contributions to $\mu_{\mathrm{CT}}$ due to CT-GS and CT-LE hybridizations are given by: ${ }^{35-37}$

$$
\begin{aligned}
\mu_{\mathrm{CT}}^{\mathrm{CT}-\mathrm{GS}} & =\frac{V_{\mathrm{CT}-\mathrm{GS}}}{\Delta E_{\mathrm{CT}-\mathrm{GS}}} \Delta \mu_{\mathrm{CT}-\mathrm{GS}} \\
\mu_{\mathrm{CT}}^{\mathrm{CT}-\mathrm{LE}} & =\frac{V_{\mathrm{CT}-\mathrm{LE}}}{\Delta E_{\mathrm{CT}-\mathrm{LE}}} \mu_{\mathrm{LE}-\mathrm{GS}}
\end{aligned}
$$

Expression (6) is the well-known Mulliken-Hush relation ${ }^{31,38-42}$ that connects the electronic coupling, $V_{\mathrm{CT}-\mathrm{GS}}$, energy gap, $\Delta E_{\mathrm{CT}-\mathrm{GS}}$, and transition dipole moment between the CT and GS states, $\mu_{\mathrm{CT}}^{\mathrm{CT}-\mathrm{GS}}$, with the difference between the dipole moments of the diabatic CT and GS states, $\Delta \mu_{\mathrm{CT}-\mathrm{GS}}$. Since the latter is defined by the electron transfer distance from $\mathrm{D}$ to $\mathrm{A}$, its value is usually much larger than those of the intra-molecular transition dipole moments, see Table 3. As a result, for similar $V / \Delta E$ ratios, the contribution due to CT-GS hybridization will be larger than that due to CT-LE coupling. Expression (6) defines the well-known intensity borrowing effect and is obtained by treating the coupling between CT and LE states as a perturbation. ${ }^{35}$ As an illustrative example, we can consider the absolute values of the transition dipole moments $\mu_{\mathrm{CT}}^{\mathrm{CT}-\mathrm{GS}}$ and $\mu_{\mathrm{CT}}^{\mathrm{CT}-\mathrm{LE}}$ in TTM- $\alpha$ PyID, which is the emitter that overall displays the largest $V_{\mathrm{CT}-\mathrm{LE}} / \Delta E_{\mathrm{CT}-\mathrm{LE}}$ hybridization coefficient between the GS state and $\mathrm{LE}_{\mathrm{r}}$ states. The $\mu_{\mathrm{CT}}^{\mathrm{CT}-\mathrm{GS}}$ value is estimated to be $3.32 \mathrm{D}$, which represents about $93 \%$ of the total $\mu_{\mathrm{CT}}$

Table 3 Total transition dipole moment of the CT state $\left(\mu_{\mathrm{CT}}\right)$, dipole moment difference between the CT and ground states ( $\Delta \mu_{\mathrm{CT}-\mathrm{GS}}$ ), values of electronic couplings, energy gaps, and transition dipole moments $(V, \Delta E$, and $\mu$ ) between the diabatic electronic states, and relative contributions to

\begin{tabular}{|c|c|c|c|c|c|}
\hline State & $\begin{array}{l}\text { TTM- } \\
1 \mathrm{Cz}\end{array}$ & $\begin{array}{l}\text { TTM- } \\
\alpha \text { PyID }\end{array}$ & $\begin{array}{l}\text { TTM- } \\
\text { ßPyID }\end{array}$ & $\begin{array}{l}\text { TTM- } \\
\gamma \text { PyID }\end{array}$ & 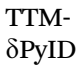 \\
\hline$\mu_{\mathrm{CT}}(\mathrm{D})$ & 3.71 & 3.55 & 3.53 & 3.31 & 3.59 \\
\hline$V_{\mathrm{CT}-\mathrm{GS}}(\mathrm{meV})$ & 314 & 435 & 336 & 342 & 337 \\
\hline$\Delta E_{\mathrm{CT}-\mathrm{GS}}(\mathrm{eV})$ & 2.19 & 2.45 & 2.33 & 2.40 & 2.30 \\
\hline$\Delta \mu_{\mathrm{CT}-\mathrm{GS}}(\mathrm{D})$ & 24.9 & 18.7 & 23.4 & 22.3 & 23.5 \\
\hline$V_{\mathrm{CT}-\mathrm{LE}_{1}}(\mathrm{meV})$ & 19 & 27 & 20 & 20 & 19 \\
\hline$\Delta E_{\mathrm{CT}-\mathrm{LE}}(\mathrm{eV})$ & 0.63 & 0.37 & 0.48 & 0.40 & 0.51 \\
\hline$\mu_{\mathrm{LE}_{1}-\mathrm{GS}}(\mathrm{D})$ & 1.45 & 1.28 & 1.55 & 1.57 & 1.49 \\
\hline$V_{\mathrm{CT}-\mathrm{LE}_{\mathrm{r} 1}}(\mathrm{meV})$ & 147 & 197 & 107 & 158 & 148 \\
\hline$\Delta E_{\mathrm{CT}-\mathrm{LE} \mathrm{r}_{1}}(\mathrm{eV})$ & 1.12 & 0.92 & 1.00 & 0.93 & 1.02 \\
\hline$\mu_{\mathrm{LE}_{\mathrm{r} 1}-\mathrm{GS}}(\mathrm{D})$ & 3.68 & 4.18 & 3.11 & 3.58 & 3.43 \\
\hline$V_{\mathrm{CT}-\mathrm{LE}_{\mathrm{r} 2}}(\mathrm{meV})$ & 66 & 104 & 81 & 181 & 67 \\
\hline$\Delta E_{\mathrm{CT}-\mathrm{LE} E_{\mathrm{r} 2}}(\mathrm{eV})$ & 1.30 & 1.04 & 1.18 & 1.12 & 1.20 \\
\hline$\mu_{\mathrm{LE}_{\mathrm{r} 2}-\mathrm{GS}}(\mathrm{D})$ & 3.96 & 3.95 & 3.91 & 3.75 & 3.95 \\
\hline$\mu_{\mathrm{CT}}^{\mathrm{CT}-\mathrm{GS}}(\mathrm{D})$ & 3.57 & 3.32 & 3.37 & 3.18 & 3.44 \\
\hline$\mu_{\mathrm{CT}}^{\mathrm{CT}-\mathrm{LE}_{1}}(\mathrm{D})$ & 0.04 & 0.09 & 0.06 & 0.08 & 0.06 \\
\hline$\mu_{\mathrm{CT}}^{\mathrm{CT}-\mathrm{LE}_{\mathrm{r} 1}}(\mathrm{D})$ & 0.48 & 0.90 & 0.33 & 0.61 & 0.50 \\
\hline$\mu_{\mathrm{CT}}^{\mathrm{CT}-\mathrm{LE}_{\mathrm{r} 2}}$ (D) & 0.20 & 0.40 & 0.27 & 0.61 & 0.22 \\
\hline
\end{tabular}
$\mu_{\mathrm{CT}}$ due to hybridization $\left(\mu_{\mathrm{CT}}^{\mathrm{A}}\right)^{\mathrm{B}}$ ) in the TTM-1Cz and TTM-xPyID radicals value $(3.55 \mathrm{D})$. The $\mu_{\mathrm{CT}}^{\mathrm{CT}-\mathrm{LE}}$ values due to the $\mathrm{LE}_{\mathrm{r} 1}$ and $\mathrm{LE}_{\mathrm{r} 2}$ states are equal to $0.9 \mathrm{D}$ and $0.4 \mathrm{D}$, respectively. In order to appreciate the individual contributions to the total $\mu_{\mathrm{CT}}$ value, since the transition dipole moments are vectors, their actual orientations must be taken into account. For instance, in all molecules, $\mu_{\mathrm{CT}}^{\mathrm{CT}-\mathrm{LE}_{\mathrm{r} 1}}$ is antiparallel to the DFT-derived $\mu_{\mathrm{CT}}$ vector $\left(\mu_{\mathrm{CT}}^{\mathrm{CT}-\mathrm{GS}}\right)$ while $\mu_{\mathrm{CT}}^{\mathrm{CT}-\mathrm{LE}_{\mathrm{r} 2}}$ is nearly perpendicular to both $\mu_{\mathrm{CT}}^{\mathrm{CT}-\mathrm{LE}_{\mathrm{rl}}}$ and $\mu_{\mathrm{CT}}^{\mathrm{CT}-\mathrm{GS}}$ (see Fig. S3, ESI $\dagger$ ). Although an exact assessment of how much the LE states contribute to the radiative decay would require to compute the $\mu_{\mathrm{CT}}^{\mathrm{CT}-\mathrm{LE}}$ contributions from all LE states, our study unambiguously demonstrates that the $\mu_{\mathrm{CT}}$ values and consequently the radiative rates in the TTM- $1 \mathrm{Cz}$ and TTM-xPyID radicals are dominated by the interactions between the CT and ground states.

We now turn to a discussion of the nonradiative transitions from the CT state to the ground state. We start with the decay rate, $k_{\mathrm{nr}}^{\mathrm{CT}-\mathrm{GS}}$, arising from the CT-GS electronic coupling. The DFTcalculated electronic couplings, $V_{\mathrm{CT}-\mathrm{GS}}$, adiabatic CT energies, $E_{\mathrm{CT}}^{\mathrm{a}}$, and reorganization energies, $\lambda$, of all radicals are collected in Table S3 (ESI $\dagger$ ). We note that, as shown in Table S4 (ESI $\dagger$ ), the $k_{\mathrm{nr}}^{\mathrm{CT}-\mathrm{GS}}$ values strongly depend on the way $\lambda$ is partitioned into classical $\left(\lambda_{\mathrm{c}}\right)$ and quantum $\left(\lambda_{\mathrm{qm}}\right)$ contributions. In order to gain a better understanding, we also computed the geometry relaxation energies of the TTM and donor (isolated) fragments occurring as a result of reduction and oxidation, respectively; the results are collected in Table S5 (ESI $\dagger$ ). The sum of these two relaxation energies provides a good estimate of the intra-molecular contribution to the CT-state relaxation. The results show that, in the case of TTM-1Cz, about $65 \%$ of the $\lambda$ value obtained from the CT-state geometry optimization can be attributed to $\lambda_{\mathrm{qm}}$. In the case of the TTM-xPyID radicals, the contribution from $\lambda_{\mathrm{qm}}$ increases to $\sim 75 \%$, except in the TTM- $\gamma$ PyID case where this value goes up to $85 \%$ (which is due to an increase of about $60 \mathrm{meV}$ in the relaxation energy of the $\gamma$ PyID fragment in comparison to the other xPyID donors, a feature likely associated with the para-type interactions between the two nitrogens in this derivative).

The computed $k_{\mathrm{nr}}^{\mathrm{CT}-\mathrm{GS}}$ values based on the DFT-derived parameters are collected in Table 4 . The results show that the $k_{\mathrm{nr}}^{\mathrm{CT}-\mathrm{GS}}$ values of the TTM-1Cz and TTM- $\gamma$ PyID molecules are much larger than in the other systems. In the case of TTM-1Cz, this is due to a relatively smaller CT-state energy; this can be related to the higher HOMO energy (lower ionization potential) of the carbazole donor in comparison to the other xPyID donors (see Fig. S1, ESI $\dagger$ ). In the case of TTM- $\gamma$ PyID, the increase in $k_{\mathrm{nr}}^{\mathrm{CT}-\mathrm{GS}}$ is coming from the larger $\lambda_{\mathrm{qm}}$ value in the CT state, which is due to an increase in the relaxation energy of the $\gamma$ PyID donor upon oxidation in comparison to the other xPyID donors and carbazole. The contributions coming from the CT-LE hybridization, $f_{\mathrm{CT}-\mathrm{LE}}^{2} k_{\mathrm{nr}}^{\mathrm{LE}-\mathrm{GS}}$, to the non-radiative rate are of the same order as $k_{\mathrm{nr}}^{\mathrm{CT}-\mathrm{GS}}$ in the case of TTM- $\alpha$ PyID, TTM- $\beta$ PyID, and TTM- $\delta$ PyID. As a result, the CT-LE hybridization plays a marginal role in the $k_{\mathrm{nr}}$ value of TTM-1Cz and TTM- $\gamma$ PyID while it is an important factor to consider in the other systems.

It is worth recalling that the experimental rates strongly depend on the measurement conditions. For instance, the rates 
Table 4 Calculated values of the nonradiative decay rates $\left(k_{\mathrm{nr}}\right)$ along with their contributions due to CT-GS hybridization ( $k_{\mathrm{nr}}^{\mathrm{CT}}$-GS) and CT-LE hybridization $\left(f_{\mathrm{CT}-\mathrm{LE}}^{2} f_{\mathrm{nr}}^{\mathrm{LE}-\mathrm{GS}}\right)$ in the TTM-1Cz and TTM-xPyID radicals. The experimental values measured in cyclohexane and toluene are also shown for the sake of comparison

\begin{tabular}{|c|c|c|c|c|c|}
\hline State & $k_{\mathrm{nr}}^{\mathrm{CT}-\mathrm{GS}} \times 10^{7} \mathrm{~s}^{-1}$ & $f_{\mathrm{CT}-\mathrm{LE}}^{2} k_{\mathrm{nr}}^{\mathrm{LE}-\mathrm{GS}} \times 10^{7} \mathrm{~s}^{-1}$ & $k_{\mathrm{nr}} \times 10^{7} \mathrm{~s}^{-1}$ & $k_{\mathrm{nr}} \times 10^{7} \mathrm{~s}^{-1}(\exp )^{a}$ & $k_{\mathrm{nr}} \times 10^{7} \mathrm{~s}^{-1}(\exp )^{b}$ \\
\hline TTM-1Cz & 2.56 & 0.02 & 2.57 & 1.13 & 3.21 \\
\hline TTM- $\alpha$ PyID & 0.07 & 0.09 & 0.16 & 0.90 & 0.33 \\
\hline TTM- $\beta$ PyID & 0.07 & 0.03 & 0.10 & 0.04 & 0.14 \\
\hline TTM- $\gamma$ PyID & 1.80 & 0.04 & 1.84 & 1.71 & 1.81 \\
\hline TTM- $\delta$ PyID & 0.17 & 0.02 & 0.20 & 0.26 & 0.12 \\
\hline
\end{tabular}

${ }^{a}$ Measured in cyclohexane. ${ }^{b}$ Measured in toluene. ${ }^{17}$

for TTM- $\alpha$ PyID, TTM- $\beta$ PyID, and TTM- $\delta$ PyID substantially differ when measured in cyclohexane while they are very similar in toluene, see Table 4 . Overall, the experimental data point to $k_{\mathrm{nr}}$ rates a few times faster in TTM- $1 \mathrm{Cz}$ and TTM- $\gamma$ PyID compared to TTM- $\alpha$ PyID, TTM- $\beta$ PyID, and TTM- $\delta$ PyID, a trend that our calculations reproduce very well.

\section{Conclusions}

We have performed long-range corrected density functional theory calculations in order to evaluate the electronic structure and luminescence properties in donor-acceptor $\left(\mathrm{D}^{-} \mathrm{A}^{\bullet}\right)$ radicals based on the combination of a pyridoindole (xPyID) or carbazole donor and a trichlorotriphenylmethyl (TTM ${ }^{\bullet}$ ) acceptor. We were especially interested in understanding the impact of hybridization between charge-transfer (CT) and local-excitation (LE) states on the radical emissive properties. Our calculations show that the electronic structures of the TTM- $1 \mathrm{Cz}$ and TTM-xPyID radicals investigated here follow the Aufbau principle. The introduction of an additional nitrogen atom into carbazole to form pyridoindole leads to a slight increase in the energies of the lowest doublet excited states while the variation in the $\mathrm{D}_{1}$ energy as a function of the nitrogen position within the pyridoindole moiety is small, which is consistent with experiment.

The $\mathrm{D}_{1}$ state for all TTM-based $\mathrm{D}^{-\mathrm{A}^{\bullet}}$ radicals has a CT nature, which arises from electron transfer from the $\mathrm{Cz}$ or xPyID donor to the TTM acceptor; adding the donor moiety leads to an increase in the radiative rates by 3 to 6 times $v s$. the (isolated) TTM radical. The computed radiative rates for the TTM-xPyID radicals are similar to that of TTM-1Cz. As analysis of the relative contributions of the CT-GS and CT-LE hybridizations to the transition dipole moments underscores that the radiative rates in these radicals are mainly dominated by the electronic couplings between the CT state and the ground state even though the high-energy LE states can have a significant CT component.

The chemical modification of the donor unit from carbazole to pyridoindole generally leads to a decrease in the nonradiative decay rates due to the increased energy of the CT state; an exception is the TTM- $\gamma$ PyID radical in which the increase in the relaxation energy of the $\gamma$ PyID donor upon oxidation results in a significant increase in the nonradiative rate. This result highlights the major impact that electron-vibrational interactions have on nonradiative transitions and the need to select donor moieties with very small relaxation energies; this is usually a characteristic of large-size, fully conjugated rigid molecules.

We find that the impact of the CT-LE hybridization on the nonradiative rates is moderate for TTM- $1 \mathrm{Cz}$ and TTM- $\gamma$ PyID while it plays an important role in the case of the other TTM-xPyID radicals. The negative effect of the CT-LE hybridization on nonradiative transitions is expected to increase for TTM-D radicals with CT states approaching the $\mathrm{D}_{1}$ state of the TTM. Therefore, the non-emissive $\mathrm{D}_{1}$ state of TTM and PTM represents an intrinsic limitation to achieve efficient highenergy emitters based on a $\mathrm{D}-\mathrm{A}^{\bullet}$ motif involving the TTM or PTM radical cores.

We also note that, while the $V_{\mathrm{CT}-\mathrm{GS}}$ and $V_{\mathrm{CT} \text {-LE }}$ electronic couplings have a strong impact on both radiative and nonradiative rates, their impact on the emitter PLQY [PLQY = $\left.k_{\mathrm{r}} /\left(k_{\mathrm{r}}+k_{\mathrm{nr}}\right)\right]$ is expected to be less significant. For instance, in the absence of CT-LE hybridization, according to eqn (1), (2), and (5) the PLQY is independent of the electronic coupling and instead is determined by $\Delta \mu_{\mathrm{CT}-\mathrm{GS}}$. An increase in $\Delta \mu_{\mathrm{CT}-\mathrm{GS}}$, which can be obtained by increasing the effective distance between the D and A fragments, is predicted to enhance the PLQY. In addition, the increase in D-A distance should also reduce $V_{\mathrm{CT}-\mathrm{LE}}$ and consequently minimize the negative impact of the CT-LE hybridization on nonradiative transitions. To the best of our knowledge, such a design strategy for $\mathrm{D}-\mathrm{A}^{\bullet}$ radicals has not been tested yet.

Finally, a major point that follows from our study is that, in order to accurately describe the radiative and non-radiative transitions of the $\mathrm{D}^{-} \mathrm{A}^{\bullet}$ radicals, it is essential to take into account the hybridizations between both the charge-transfer and ground states (CT-GS hybridization) and the local-excitation and charge-transfer states (LE-CT hybridization).

\section{Conflicts of interest}

There are no conflicts to declare.

\section{Acknowledgements}

This work was funded by the College of Science of the University of Arizona. The authors acknowledge the use of the computing facilities of the Partnership for an Advanced Computing Environment (PACE) at the Georgia Institute of Technology and the assistance of the PACE team. 


\section{References}

1 M. Mas-Torrent, N. Crivillers, V. Mugnaini, I. Ratera, C. Rovira and J. Veciana, Organic Radicals on Surfaces: Towards Molecular Spintronics, J. Mater. Chem., 2009, 19, 1691-1695.

2 S. Kumar, Y. Kumar, S. K. Keshri and P. Mukhopadhyay, Recent Advances in Organic Radicals and Their Magnetism, Magnetochemistry, 2016, 2, 42.

3 R. Gaudenzi, J. de Bruijckere, D. Reta, I. D. P. R. Moreira, C. Rovira, J. Veciana, H. S. J. van der Zant and E. Burzurí, Redox-Induced Gating of the Exchange Interactions in a Single Organic Diradical, ACS Nano, 2017, 11, 5879-5883.

4 A. Gaita-Ariño, F. Luis, S. Hill and E. Coronado, Molecular Spins for Quantum Computation, Nat. Chem., 2019, 11, 301-309.

5 K. Kato and A. Osuka, Platforms for Stable Carbon-Centered Radicals, Angew. Chem., Int. Ed., 2019, 58, 8978-8986.

6 A. Dragulescu-Andrasi, A. S. Filatov, R. T. Oakley, X. Li, K. Lekin, A. Huq, C. Pak, S. M. Greer, J. McKay, M. Jo, J. Lengyel, I. Hung, E. Maradzike, A. E. DePrince, S. A. Stoian, S. Hill, Y.-Y. Hu and M. Shatruk, Radical Dimerization in a Plastic Organic Crystal Leads to Structural and Magnetic Bistability with Wide Thermal Hysteresis, J. Am. Chem. Soc., 2019, 141, 17989-17994.

7 V. Diez-Cabanes, D. C. Morales, M. Souto, M. Paradinas, F. Delchiaro, A. Painelli, C. Ocal, D. Cornil, J. Cornil, J. Veciana and I. Ratera, Effect of the Molecular Polarizability of SAMs on the Work Function Modification of Gold: Closed-versus Open-Shell Donor-Acceptor SAMs, Adv. Mater. Technol., 2019, 4, 1800152.

8 L. Ji, J. Q. Shi, J. Wei, T. Yu and W. Huang, Air-Stable Organic Radicals: New-Generation Materials for Flexible Electronics?, Adv. Mater., 2020, 32, 1908015.

9 V. Gamero, D. Velasco, S. Latorre, F. López-Calahorra, E. Brillas and L. Juliá, [4-(N-Carbazolyl)-2,6-dichlorophenyl]bis(2,4,6-trichlorophenyl)methyl Radical an Efficient Red Light-Emitting Paramagnetic Molecule, Tetrahedron Lett., 2006, 47, 2305-2309.

10 A. Heckmann, S. Dümmler, J. Pauli, M. Margraf, J. Köhler, D. Stich, C. Lambert, I. Fischer and U. Resch-Genger, Highly Fluorescent Open-Shell NIR Dyes: The Time-Dependence of Back Electron Transfer in Triarylamine-Perchlorotriphenylmethyl Radicals, J. Phys. Chem. C, 2009, 113, 20958-20966.

11 Q. Peng, A. Obolda, M. Zhang and F. Li, Organic Light-Emitting Diodes Using a Neutral $\pi$ Radical as Emitter: The Emission from a Doublet, Angew. Chem., Int. Ed., 2015, 54, 7091-7095.

12 A. Obolda, M. Zhang and F. Li, Evolution of Emission Manners of Organic Light-Emitting Diodes: From Emission of Singlet Exciton to Emission of Doublet Exciton, Chin. Chem. Lett., 2016, 27, 1345-1349.

13 J. Guo, X.-L. Li, H. Nie, W. Luo, S. Gan, S. Hu, R. Hu, A. Qin, Z. Zhao, S.-J. Su and B. Z. Tang, Achieving High-Performance Nondoped OLEDs with Extremely Small Efficiency Roll-Off by Combining Aggregation-Induced Emission and Thermally Activated Delayed Fluorescence, Adv. Funct. Mater., 2017, 27, 1606458.

14 X. Ai, E. W. Evans, S. Dong, A. J. Gillett, H. Guo, Y. Chen, T. J. H. Hele, R. H. Friend and F. Li, Efficient Radical-based
Light-Emitting Diodes with Doublet Emission, Nature, 2018, 563, 536-540.

15 H. Guo, Q. Peng, X.-K. Chen, Q. Gu, S. Dong, E. W. Evans, A. J. Gillett, X. Ai, M. Zhang, D. Credgington, V. Coropceanu, R. H. Friend, J.-L. Brédas and F. Li, High Stability and Luminescence Efficiency in Donor-Acceptor Neutral Radicals not Following the Aufbau Principle, Nat. Mater., 2019, 18, 977-984.

16 H. Abroshan, V. Coropceanu and J.-L. Brédas, Radiative and Nonradiative Recombinations in Organic Radical Emitters: The Effect of Guest-Host Interactions, Adv. Funct. Mater., 2020, 30, 2002916.

17 A. Abdurahman, T. J. H. Hele, Q. Gu, J. Zhang, Q. Peng, M. Zhang, R. H. Friend, F. Li and E. W. Evans, Understanding the Luminescent Nature of Organic Radicals for Efficient Doublet Emitters and Pure-Red Light-Emitting Diodes, Nat. Mater., 2020, 19, 1224-1229.

18 Z. Cui, A. Abdurahman, X. Ai and F. Li, Stable Luminescent Radicals and Radical-Based LEDs with Doublet Emission, CCS Chem., 2020, 2, 1129-1145.

19 E. Cho, V. Coropceanu and J.-L. Brédas, Organic Neutral Radical Emitters: Impact of Chemical Substitution and Electronic-State Hybridization on the Luminescence Properties, J. Am. Chem. Soc., 2020, 142, 17782-17786.

20 D. Velasco, S. Castellanos, M. López, F. López-Calahorra, E. Brillas and L. Juliá, Red Organic Light-Emitting Radical Adducts of Carbazole and Tris(2,4,6-trichlorotriphenyl)methyl Radical That Exhibit High Thermal Stability and Electrochemical Amphotericity, J. Org. Chem., 2007, 72, 7523-7532.

21 V. Diez-Cabanes, G. Seber, C. Franco, F. Bejarano, N. Crivillers, M. Mas-Torrent, J. Veciana, C. Rovira and J. Cornil, Design of Perchlorotriphenylmethyl (PTM) Radical-Based Compounds for Optoelectronic Applications: The Role of Orbital Delocalization, ChemPhysChem, 2018, 19, 2572-2578.

22 Z. Cui, S. Ye, L. Wang, H. Guo, A. Obolda, S. Dong, Y. Chen, X. Ai, A. Abdurahman, M. Zhang, L. Wang and F. Li, RadicalBased Organic Light-Emitting Diodes with Maximum External Quantum Efficiency of 10.6\%, J. Phys. Chem. Lett., 2018, 9, 6644-6648.

23 A. Abdurahman, Y. Chen, X. Ai, O. Ablikim, Y. Gao, S. Dong, B. Li, B. Yang, M. Zhang and F. Li, A Pure Red Luminescent $\beta$-Carboline-Substituted Biphenylmethyl Radical: Photophysics, Stability and OLEDs, J. Mater. Chem. C, 2018, 6, 11248-11254.

24 E. Cho, V. Coropceanu and J.-L. Brédas, Electronic Structure of Multicomponent Organic Molecular Materials: Evaluation of Range-Separated Hybrid Functionals, J. Chem. Theory Comput., 2020, 16, 3712-3719.

25 S. Hirata and M. Head-Gordon, Time-Dependent Density Functional Theory within the Tamm-Dancoff Approximation, Chem. Phys. Lett., 1999, 314, 291-299.

26 M. J. Frisch, G. W. Trucks, H. B. Schlegel, G. E. Scuseria, M. A. Robb, J. R. Cheeseman, G. Scalmani, V. Barone, G. A. Petersson, H. Nakatsuji, X. Li, M. Caricato, A. V. Marenich, J. Bloino, B. G. Janesko, R. Gomperts, B. Mennucci, H. P. Hratchian, J. V. Ortiz, A. F. Izmaylov, J. L. Sonnenberg, 
D. Williams-Young, F. Ding, F. Lipparini, F. Egidi, J. Goings, B. Peng, A. Petrone, T. Henderson, D. Ranasinghe, V. G. Zakrzewski, J. Gao, N. Rega, G. Zheng, W. Liang, M. Hada, M. Ehara, K. Toyota, R. Fukuda, J. Hasegawa, M. Ishida, T. Nakajima, Y. Honda, O. Kitao, H. Nakai, T. Vreven, K. Throssell, J. A. Montgomery, J. E. Peralta, F. Ogliaro, M. J. Bearpark, J. J. Heyd, E. N. Brothers, K. N. Kudin, V. N. Staroverov, T. A. Keith, R. Kobayashi, J. Normand, K. Raghavachari, A. P. Rendell, J. C. Burant, S. S. Iyengar, J. Tomasi, M. Cossi, J. M. Millam, M. Klene, C. Adamo, R. Cammi, J. W. Ochterski, R. L. Martin, K. Morokuma, O. Farkas, J. B. Foresman and D. J. Fox, Gaussian 16, Revision B.01, Gaussian, Inc., Wallingford CT, 2016.

27 R. C. Hilborn, Einstein Coefficients, Cross Sections, f Values, Dipole Moments, and All that, Am. J. Phys., 1982, 50, 982-986.

28 D. Toptygin, Effects of the Solvent Refractive Index and Its Dispersion on the Radiative Decay Rate and Extinction Coefficient of a Fluorescent Solute, J. Fluoresc., 2003, 13, 201-219.

29 V. Coropceanu, X.-K. Chen, T. Wang, Z. Zheng and J.-L. Brédas, Charge-Transfer Electronic States in Organic Solar Cells, Nat. Rev. Mater., 2019, 4, 689-707.

30 V. Coropceanu, J. Cornil, D. A. da Silva Filho, Y. Olivier, R. Silbey and J.-L. Brédas, Charge Transport in Organic Semiconductors, Chem. Rev., 2007, 107, 926-952.

31 R. J. Cave and M. D. Newton, Generalization of the MullikenHush Treatment for the Calculation of Electron Transfer Matrix Elements, Chem. Phys. Lett., 1996, 249, 15-19.

32 Y. Shao, Z. Gan, E. Epifanovsky, A. T. B. Gilbert, M. Wormit, J. Kussmann, A. W. Lange, A. Behn, J. Deng, X. Feng, D. Ghosh, M. Goldey, P. R. Horn, L. D. Jacobson, I. Kaliman, R. Z. Khaliullin, T. Kuś, A. Landau, J. Liu, E. I. Proynov, Y. M. Rhee, R. M. Richard, M. A. Rohrdanz, R. P. Steele, E. J. Sundstrom, H. L. Woodcock, P. M. Zimmerman, D. Zuev, B. Albrecht, E. Alguire, B. Austin, G. J. O. Beran, Y. A. Bernard, E. Berquist, K. Brandhorst, K. B. Bravaya, S. T. Brown, D. Casanova, C.-M. Chang, Y. Chen, S. H. Chien, K. D. Closser, D. L. Crittenden, M. Diedenhofen, R. A. DiStasio, H. Do, A. D. Dutoi, R. G. Edgar, S. Fatehi, L. Fusti-Molnar, A. Ghysels, A. Golubeva-Zadorozhnaya, J. Gomes, M. W. D. Hanson-Heine, P. H. P. Harbach, A. W. Hauser, E. G. Hohenstein, Z. C. Holden, T.-C. Jagau, H. Ji, B. Kaduk, K. Khistyaev, J. Kim, J. Kim, R. A. King, P. Klunzinger, D. Kosenkov, T. Kowalczyk, C. M. Krauter, K. U. Lao, A. D. Laurent, K. V. Lawler, S. V. Levchenko, C. Y. Lin, F. Liu, E. Livshits, R. C. Lochan, A. Luenser, P. Manohar, S. F. Manzer, S.-P. Mao, N. Mardirossian, A. V. Marenich,
S. A. Maurer, N. J. Mayhall, E. Neuscamman, C. M. Oana, R. Olivares-Amaya, D. P. O'Neill, J. A. Parkhill, T. M. Perrine, R. Peverati, A. Prociuk, D. R. Rehn, E. Rosta, N. J. Russ, S. M. Sharada, S. Sharma, D. W. Small, A. Sodt, T. Stein, D. Stück, Y.-C. Su, A. J. W. Thom, T. Tsuchimochi, V. Vanovschi, L. Vogt, O. Vydrov, T. Wang, M. A. Watson, J. Wenzel, A. White, C. F. Williams, J. Yang, S. Yeganeh, S. R. Yost, Z.-Q. You, I. Y. Zhang, X. Zhang, Y. Zhao, B. R. Brooks, G. K. L. Chan, D. M. Chipman, C. J. Cramer, W. A. Goddard, M. S. Gordon, W. J. Hehre, A. Klamt, H. F. Schaefer, M. W. Schmidt, C. D. Sherrill, D. G. Truhlar, A. Warshel, X. Xu, A. Aspuru-Guzik, R. Baer, A. T. Bell, N. A. Besley, J.-D. Chai, A. Dreuw, B. D. Dunietz, T. R. Furlani, S. R. Gwaltney, C.-P. Hsu, Y. Jung, J. Kong, D. S. Lambrecht, W. Liang, C. Ochsenfeld, V. A. Rassolov, L. V. Slipchenko, J. E. Subotnik, T. Van Voorhis, J. M. Herbert, A. I. Krylov, P. M. W. Gill and M. Head-Gordon, Advances in Molecular Quantum Chemistry Contained in the Q-Chem 4 Program Package, Mol. Phys., 2015, 113, 184-215.

33 N. M. O'Boyle, A. L. Tenderholt and K. M. Langner, Cclib: A Library for Package-Independent Computational Chemistry Algorithms, J. Comput. Chem., 2008, 29, 839-845.

34 I. R. Gould and S. Farid, Radiationless Decay in Exciplexes with Variable Charge Transfer, J. Phys. Chem. B, 2007, 111, 6782-6787.

35 M. Bixon, J. Jortner and J. W. Verhoeven, Lifetimes for Radiative Charge Recombination in Donor-Acceptor Molecules, J. Am. Chem. Soc., 1994, 116, 7349-7355.

36 R. J. Kokes and P. H. Emmett, The Role of Hydrogen in Raney Nickel Catalysts, J. Am. Chem. Soc., 1959, 81, 5032-5037.

37 M. D. Newton, Quantum Chemical Probes of ElectronTransfer Kinetics: the Nature of Donor-Acceptor Interactions, Chem. Rev., 1991, 91, 767-792.

38 N. S. Hush, Progress in Inorganic Chemistry, John Wiley \& Sons, 1967.

39 R. A. Marcus, Relation between Charge Transfer Absorption and Fluorescence Spectra and the Inverted Region, J. Chem. Phys., 1989, 93, 3078-3086.

40 N. S. Hush, Homogeneous and Heterogeneous Optical and Thermal Electron Transfer, Electrochim. Acta, 1968, 13, 1005-1023.

41 R. S. Mulliken, Molecular Compounds and their Spectra. II, J. Am. Chem. Soc., 1952, 74, 811-824.

42 C. Creutz, M. D. Newton and N. Sutin, Metal-Lingad and Metal-Metal Coupling Elements, J. Photochem. Photobiol., A, 1994, 82, 47-59. 